\title{
Dimensão Aplicada na Análise do Comportamento
}

\author{
Silvia Canaan-Oliveira ${ }^{12}$ \\ Universidade Federal do Pará
}

\begin{abstract}
Resumo
O presente estudo teve como objetivo examinar a lógica do uso do termo "aplicada" na Análise do Comportamento a partir de elementos identificados na literatura desta área e na linguagem cotidiana. As concepções de aplicação encontradas foram categorizadas em três grupos: a) aplicação enquanto prestação de serviço que leva a discussão para o continuum prestação de serviço X pesquisa, assemelhando-se à forma como o termo "aplicada" é utilizado na linguagem cotidiana, b) aplicação enquanto uma forma de pesquisa que revela uma revisão do conceito de aplicação na linguagem cotidiana e sua estensão para atividades científicas, direcionando a discussão para o continuum pesquisa básica x pesquisa aplicada e c) aplicação enquanto atividade simultânea de pesquisa e prestação de serviço que sinaliza o caráter complementar destas atividades. Portanto, existem tradições coexistentes na Análise do Comportamento que apoiam diferentes usos do termo "aplicada".

Palavras-chave: Análise do comportamento; aplicação; prestação de serviço; pesquisa básica e aplicada.
\end{abstract}

\section{The Applied Dimension in Behavior Analysis}

\begin{abstract}
The present study aimed to exam the logic of the term "applied" in Behavior Analysis, as found in the literature in this area and in everyday language. The conceptions of application were categorized in three groups: a) application as service rendering, leading the discussion to the continum service rendering $\mathrm{x}$ research, which is similar to everyday language use, b) application as a research mode, a revision of the concept of application in everyday language and its extension to scientific activities, turning the discussion to the continuum basic $\mathrm{X}$ applied research and c) application as simultaneous activities of research and service rendering which indicates the complementary character of these activities. Therefore, there are coexistent traditions in Behavior Analysis that support different uses of the term "applied".

Keywords: Behavior analysis; application; service rendering; basic and applied research.
\end{abstract}

Nas últimas décadas, a Análise Comportamental Aplicada tem sido alvo de freqüentes discussões quanto aos seus objetivos e práticas, bem como sobre os tipos de atividades nas quais os analistas aplicados do comportamento devem ou não se envolver (Baer, Wolf \& Risley, 1968; Birnbrauer, 1979; Deitz, 1978; Hayes, 1998; Hayes, Rincover \& Solnick, 1980; Johnston, 1996; Luna, 1997; Pierce \& Epling, 1980; Poling, Picker \& Grossett, 1981). Embora sejam vários os temas principais das discussões, na maioria delas acabam ocorrendo reflexões sobre o significado da palavra "aplicada" talvez por esta constituir o termo que, ao ser acrescentado à expressão já conhecida Análise do Comportamento, traz-lhe alguns problemas.

Segundo o Novo Dicionário da Língua Portuguesa (Ferreira, 1986), o verbo "aplicar" significa sobrepor, pôr em prática, empregar, adaptar, adequar. Neste sentido, o adjetivo "aplicado(a)" se refere a algo que foi sobreposto, posto em prática ou empregado. Considerando os dois últimos sentidos do verbo "aplicar" citados acima, especulase que o processo de aplicação pode envolver também algumas adaptações ou adequações. Portanto, o uso da

\footnotetext{
${ }^{1}$ Endereço para correspondência: Av. Nazaré, 568 / 901, 66035170. Belém, Pará. Fone: (91) 2492412 / 224 0196. E-mail: canaan.bel@zaz.com.br ${ }^{2}$ Trabalho parcialmente financiado pela CAPES (PICDT).
}

palavra "aplicada" na linguagem cotidiana parece implicar numa separação entre dois mundos: um mundo que produz conhecimento e um mundo onde o conhecimento é aplicado na prática.

Concepções de aplicação encontradas em alguns trabalhos na área da Análise do Comportamento serão examinadas a seguir como exemplos do que os analistas do comportamento têm dito sobre a dimensão aplicada. Tais concepções permitirão uma reflexão acerca da lógica do uso do termo "aplicada" na linguagem técnica da Análise do Comportamento e o levantamento de algumas questões sobre este tema.

\section{Aplicação Enquanto Prestação de Serviço}

Alguns analistas do comportamento (Azrin, 1977; Johnston, 1996; Luna, 1997) utilizam a palavra "aplicada" no sentido de prestação de serviço, concebendo o trabalho aplicado como intimamente ligado à psicologia profissional. Azrin (1977), por exemplo, utiliza o termo em questão neste sentido quando afirma: "Mas curas são o que a psicologia aplicada tem como seu domínio" (p. 141). Luna (1997) também trata deste assunto ao afirmar que, nas atividades de prestação de serviço, o profissional depara-se com interlocutores (indivíduo, grupo, comunidade) que necessitam de seu auxílio para resolver seus problemas; 
cabe ao profissional identificar tais problemas (ou levar seu cliente a fazê-lo) e colocar sua competência a serviço do encaminhamento de soluções.

A concepção de aplicação como prestação de um serviço profissional é também partilhada por Johnston (1996) que afirma o seguinte:

a prioridade da prática aplicada é prestar um serviço eficaz e não responder questões experimentais. As únicas questões que os prestadores de serviço deveriam rotineiramente enfrentar dizem respeito à avaliação do problema atual, seleção de procedimentos apropriados a partir da tecnologia disponível, adaptação de procedimentos para circunstâncias locais, administração de procedimentos consistentes com exigências tecnológicas, e acompanhamento dos resultados. (pp. 43-44)

Portanto, o uso da palavra "aplicada" no sentido de prestação de serviço parece implicar na adoção de um critério de relevância social já que as atividades aplicadas em geral são conduzidas visando a solução ou cura de problemas sociais. Neste sentido, considera-se uma aplicação como bem sucedida quando um profissional consegue auxiliar o cliente a resolver seus problemas.

A concepção de aplicação como exclusiva prestação de serviço implica no tratamento desta questão em contraposição à noção de ciência. A não-cientificidade da aplicação foi ressaltada por Johnston (1996) ao afirmar que "... a prioridade da prática aplicada é prestar um serviço eficaz e não responder a questões experimentais" (p. 43). Em um outro momento, este autor amplia sua argumentação dizendo o seguinte:

assim como ocorre na aplicação da engenharia e da tecnologia médica, a prática não é geralmente uma ocasião para descoberta, compreensão ou explicação em um sentido científico. Embora prestadores de serviço individuais possam adquirir experiência desta maneira, tal conhecimento é bem diferente do que o obtido por pesquisadores e não pode substituir a pesquisa aplicada. (p. 44)

Em resumo, o uso da palavra "aplicada" enquanto prestação de serviço encontra-se bem estabelecido na literatura comportamental. Este uso do termo em questão parece ser semelhante ao modo como ele é empregado na linguagem cotidiana, ou seja, como uma atividade de prestação de serviço que aplica ou deveria aplicar um conhecimento que foi adquirido em um outro lugar na busca de soluções para um problema de natureza prática.

\section{Aplicação Enquanto uma Forma de Pesquisa}

O conceito de "aplicação" tal como concebido na linguagem cotidiana parece ter sido revisado pela Análise do Comportamento através de sua extensão para atividades ditas científicas. Deitz (1983), por exemplo, afirma que a palavra "aplicada" é "utilizada por alguns autores para classificar aquele tipo de atividade científica que busca identificar as variáveis responsáveis não apenas por qualquer comportamento, mas por comportamento socialmente relevante" (p. 105). Este critério de relevância social é também utilizado por Baer e colaboradores (1968) ao definir pesquisa aplicada como aquela cujas decisões metodológicas quanto à seleção de sujeitos, comportamentos, estímulos e procedimentos são determinadas pelo interesse imediato que a sociedade demonstra nos problemas sendo estudados ao invés de sua relevância para a teoria. Assim, uma análise comportamental aplicada é uma forma de pesquisa que é notavelmente diferente de qualquer outra forma de pesquisa apenas pelo "interesse que a sociedade demonstra no problema sendo estudado" (Baer \& cols., 1968, p. 92).

Alguns autores (Baer \& cols., 1968; Deitz, 1983; Hayes, 1998; Johnston, 1996) tratam do conceito de aplicação enquanto uma forma de pesquisa através de sua comparação com outras atividades científicas consideradas básicas ou não-aplicadas. Hayes (1998), por exemplo, ao considerar o contexto da terapia comportamental, afirma:

alguma preocupação é expressa pela falta de coerência teórica na terapia comportamental ou em campos aplicados afins e uma necessidade em atender aos princípios teóricos identificados pela ciência básica. Este tipo de preocupação é visto usualmente como um assunto de ciência básica versus ciência aplicada. (p. 4)

Para Baer e colaboradores (1968), as diferenças entre pesquisa básica e aplicada não são diferenças entre aquela que "descobre" e aquela que meramente "aplica" aquilo que já é conhecido. Ambas "perguntam o que controla o comportamento sob estudo" (p. 91). Johnston (1996) também examina esta questão utilizando a concepção de ciência aplicada adotada por outras ciências naturais:

Ciência aplicada pode ser definida como pesquisa experimental que está conectada com a pesquisa básica através de seu estilo experimental e uma base em princípios fundamentais, diretamente dirigida por questões e problemas aplicados, mas não comprometida pelas limitações práticas ou pelos interesses imediatos de prestação de serviço em contextos aplicados. (p. 38)

Em resumo, o uso da palavra "aplicada" enquanto uma forma de pesquisa também se encontra bem estabelecido na literatura comportamental. Este uso parece ser diferente do modo como o termo é empregado na linguagem cotidiana já que não busca solucionar ou modificar comportamentos-problemas.

Psicologia: Reflexão e Crítica, 2003, 16(2), pp. 349-354 
Assim, a pesquisa aplicada é concebida como uma atividade científica que visa compreender o comportamento socialmente importante através da investigação das variáveis das quais o mesmo é função. $\mathrm{O}$ que diferencia a pesquisa aplicada da pesquisa básica é meramente a relevância social do comportamento em questão. Nesta concepção de aplicação enquanto uma forma de pesquisa o papel do sujeito humano dentro e fora do laboratório é valorizado pois, tradicionalmente, o ser humano era mais associado com a aplicação enquanto mera prestação de serviço.

\section{Aplicação como Pesquisa e Prestação de Serviço}

Apesar da palavra "aplicada" ser utilizada separadamente nos dois sentidos discutidos acima, alguns autores (Baer \& cols., 1968; Hake, 1982; Luna, 1997; Watson, 1913) também empregam este termo para se referir simultaneamente a atividades de pesquisa e de prestação de serviço. Watson (1913), em seu manifesto Psychology as the behaviorist viens it, é um dos primeiros a sugerir que estas áreas nem sempre se constituíram em campos separados ao afirmar o seguinte:

Pedagogia experimental, psicologia das drogas, psicologia dos testes, psicopatologia são todos crescimentos vigorosos. Elas são às vezes erroneamente chamadas psicologia "prática" ou "aplicada". Certamente não poderia haver um pior título. No futuro podem se desenvolver empreendimentos vocacionais que realmente apliquem a psicologia. No presente estes campos são verdadeiramente científicos e estão em busca de amplas generalizações que levarão ao controle do comportamento humano. (p. 169)

Então, para este autor, tanto a pesquisa psicológica quanto a psicologia profissional são consideradas como atividades científicas na medida que "estão em busca de amplas generalizações que levarão ao controle do comportamento humano" (Watson, 1913, p. 169).

Outros autores (Baer \& cols.,1968; Luna, 1997) também adotam uma concepção de aplicação envolvendo simultaneidade de atividades de pesquisa e prestação de serviço. Luna (1997), por exemplo, considera ser perfeitamente plausível o desenvolvimento de um tipo de pesquisa por terapeutas em que fenômenos simulados pelo laboratório sejam colocados à prova na situação clínica. Entretanto, pode-se afirmar que o casamento da prestação de serviço com a pesquisa foi consumado com o lançamento do Journal of Applied Behavior Analysis (JABA) e a definição de aplicação proposta por Baer e colaboradores (1968) no primeiro número do referido periódico científico. Segundo estes autores, análise comportamental aplicada é:

o processo de aplicar princípios do comportamento às vezes tentativos para a melhoria de comportamentos específicos, e simultaneamente avaliar se quaisquer modificações observadas são de fato atribuíveis ao processo de aplicação e, em caso positivo, a que partes daquele processo. (p. 91)

Então, Baer e colaboradores (1968) estabelecem as bases da relação entre pesquisa aplicada e prestação de serviço, indicando que a palavra "aplicada" também é utilizada para se referir a atividades combinadas de pesquisa e de prestação de serviço. Tal combinação sugere uma relação próxima entre pesquisa aplicada e análise experimental do comportamento já que a pesquisa aplicada precisa tanto demonstrar o controle sobre o comportamento bem como a articulação entre os procedimentos utilizados e os princípios derivados da ciência básica.

Em resumo, no campo da Análise Comportamental Aplicada, o termo "aplicada" é usado em dois sentidos claramente separados. A palavra "aplicada" é utilizada para se referir ao trabalho prático em uma área tecnológica que aplica ou deveria aplicar um conhecimento que foi amplamente desenvolvido em outro lugar; tal trabalho pode ser baseado em descobertas de pesquisa mas quem o conduz não está buscando os mesmos tipos de respostas procuradas pela pesquisa básica. A palavra "aplicada" é também empregada por alguns autores para classificar aquele tipo de atividade científica que busca identificar as variáveis responsáveis por comportamentos socialmente importantes.

Quando a palavra "aplicada" é utilizada no primeiro sentido, a argumentação é construída com base no continuum pesquisa versus prestação de serviço. $\mathrm{O}$ uso do termo "aplicada" no segundo sentido parece direcionar as discussões para o continum pesquisa básica versus pesquisa aplicada (Deitz, 1983; Hayes, 1998). Além disso, o termo aplicada pode também ser empregado para designar atividades que combinam interesses da pesquisa e da prestação de serviço.

Entretanto, alguns autores (Bennett, 1987; Birnbrauer, 1979; Deitz, 1978; Hayes \& cols., 1980; Johnston, 1996; Pierce \& Epling, 1980) têm considerado negativa a combinação da pesquisa com a prestação de serviço. Deitz (1978), por exemplo, argumentou que o campo da Análise Comportamental Aplicada está se tornando cada vez menos científico e cada vez mais tecnológico, havendo menos interesse em questões conceituais. Ou seja, a ênfase mudou da análise experimental precisa das relações funcionais entre variáveis independentes e dependentes para a efetuação de modificações em variáveis dependentes per se que possam contribuir para a melhora do comportamento. Esta conclusão é partilhada por Hayes e colaboradores (1980) que analisaram artigos publicados no JABA de 1968 a 1978. Pierce e Epling (1980) também chegaram a uma conclusão semelhante baseados numa análise de artigos publicados no 
Volume 11 do JABA. Na mesma linha de raciocínio, Bennett (1987) afirma o que tem sido visto como "uma mudança do foco na pesquisa aplicada daquele de analisar comportamentos socialmente importantes para aquele de estabelecer procedimentos tecnológicos que podem ser usados para promover satisfação do consumidor" (p. 5). Assim, a análise aplicada do comportamento parece ter dois aspectos - análise comportamental e tecnologia comportamental.

Birnbrauer (1979) considera que tem havido uma mistura excessiva dos interesses da pesquisa aplicada e da prestação de serviço na literatura comportamental, em detrimento de ambas. Johnston (1996) também se posiciona sobre esta questão afirmando o seguinte:

a concepção tradicional da análise comportamental aplicada como servindo simultaneamente a ambos os interesses pode nos impedir de ser bem sucedidos em qualquer destas atividades. As exigências de que todo estudo aplicado simultaneamente a) utilize sujeitos e contextos comuns para o problema aplicado, b) produza efeitos que sejam visíveis o bastante para resolver o problema imediato e c) examine a generalidade aplicada do procedimento freqüentemente pressupõem a pesquisa analítica necessária para desenvolver tecnologias verdadeiras. Além disso, trazer as exigências intrusivas e excessivas dos métodos de pesquisa para a prestação rotineira de serviços pode complicar também esta atividade. (p. 43)

Portanto, constata-se que, apesar da palavra aplicada ser freqüentemente utilizada para designar atividades simultâneas de pesquisa e de prestação de serviço, tal combinação parece ser problemática devido à sobreposição de duas áreas que, embora possam interagir, nem sempre são compatíveis e do reduzido grau de cientificidade de grande parte dos estudos aplicados publicados. O que ocorre na maioria dos estudos publicados é que a demonstração da capacidade para resolver um problema prático específico acaba comprometendo os objetivos ditos "científicos" tais como aprender sobre etiologia e manutenção do comportamento, elucidar fontes de controle ou compreender a contribuição de múltiplas variáveis para o fenômeno sob estudo.

Assim, é razoável afirmar que todos os sentidos em que o termo "aplicada" é empregado parecem ser corretos na medida em que existem tradições coexistentes dentro da Análise Comportamental que apoiam as diferentes definições. Pode-se concluir, então, que a palavra "aplicada" no contexto da Análise do Comportamento apresenta uma "família de significados" (Wittgenstein, 1953/1987).

\section{Considerações Finais}

A discussão acima acerca de diferentes concepções de aplicação ilustra o debate que tem havido sobre o tema e possibilita o delineamento de algumas questões que emergem de tal debate. Uma das questões mais relevantes diz respeito ao fato de que o uso da palavra "aplicada" tanto na linguagem cotidiana como na linguagem técnica da Análise do Comportamento, parece implicar numa separação entre dois mundos: um mundo que produz conhecimento e um mundo onde o conhecimento é aplicado na prática.

Ryle (1954/1993) discute as relações entre o Mundo da Ciência e o Mundo Cotidiano na seguinte citação:

Quando estamos num certo estado de espírito intelectual, parece que descobrimos choques entre as coisas que os cientistas nos dizem sobre nossos móveis, nossas roupas e nossos membros, e as coisas que nós dizemos a respeito deles. Somos capazes de expressar essas divergências sentidas dizendo que o mundo cujas peças e membros são descritos pelos cientistas é diferente do mundo cujas peças e membros nós próprios descrevemos; e, no entanto, como só pode existir um mundo, um desses pretensos mundos deve ser fictício. Além disso, como hoje em dia ninguém é suficientemente ousado para contestar a ciência, o mundo que descrevemos é que deve ser fictício. (p. 110)

Portanto, Ryle (1954/1993) indica o fato de que as pessoas costumam perceber o mundo da ciência e o mundo cotidiano como diferentes ou divergentes e que, "como só pode existir um mundo", acabam contestando e desvalorizando o mundo da vida diária. Entretanto, este autor parece reconhecer que a aparência de um antagonismo entre os diferentes modos de descrever os fenômenos é enganadora. $\mathrm{Na}$ verdade, os princípios que regem a realidade estudada pela ciência são os mesmos que regem a realidade cotidiana e, assim, os mundos dentro e fora do laboratório estão continuamente se alimentando na busca do conhecimento. Neste sentido, a ciência e a vida cotidiana se complementam na compreensão do mundo que é um só.

Skinner (1953/1985) privilegia o laboratório e os métodos experimentais enquanto elementos do "mundo da ciência" que oferecem condições ideais para uma análise científica dos processos comportamentais como demonstra a seguinte citação:

No laboratório muitas condições são simplificadas e as condições irrelevantes freqüentemente eliminadas ... É verdade que podemos obter controle sobre o comportamento somente na medida em que conseguimos controlar os fatores por ele responsáveis. O que um estudo científico faz é permitir a utilização ótima dos controles que possuímos. A simplificação 
do laboratório revela a relevância de fatores que poderíamos de outro modo deixar passar. (p. 33)

De acordo com a visão skinneriana, estes métodos tornam visíveis as relações funcionais entre o comportamento do sujeito experimental e aspectos da situação experimental, aumentando a probabilidade de que as explicações ou descrições verbais subseqüentes do pesquisador acerca destas relações fiquem diretamente sob controle de estímulo fornecido pela situação experimental. Desta forma, os métodos experimentais participam do controle exercido sobre o comportamento verbal do pesquisador.

Entretanto, Skinner (1953/1985) também identifica alguns limites do laboratório e dos métodos experimentais para o controle e predição do comportamento que ocorre em situações da vida diária ou mundo cotidiano:

Atualmente a pesquisa experimental do comportamento humano não é às vezes tão ampla quanto se poderia desejar. Nem todos os processos comportamentais são fáceis de se estabelecer no laboratório, e a precisão nas medidas é às vezes obtida às custas da irrealidade nas condições. Aqueles que se preocupam principalmente com a vida cotidiana dos indivíduos, muitas vezes se impacientam com estes artificialismos, mas na medida em que relações relevantes podem ser submetidas a controle experimental, o laboratório oferece a melhor oportunidade para obter os resultados quantitativos para uma análise científica. (p. 47)

Goldiamond (1965) também reconhece a separação existente entre o mundo do laboratório que produz conhecimento e um mundo onde o conhecimento é aplicado na prática. Ele discute esta questão ao tratar da prática clínica e afirma o seguinte:

no presente, existe muito pouca relação entre boa parte da pesquisa psicológica, seja experimental ou clínica, e a prática clínica. A distância que se desenvolveu entre pesquisa e prática é tal que as repercussões estão correntemente sendo sentidas em programas de treinamento e em organização profissional. (pp. 56-58)

Entretanto, este autor parece alinhar-se com aqueles que acreditam na complementarie-dade das atividades dos Analistas Experimentais do Comportamento e dos Analistas Comportamentais Aplicados quando, juntamente com Dyrud, afirma que:

A Análise do Comportamento em termos de suas conseqüências como uma disciplina de laboratório sistemática é, evidentemente, atualmente a preocupação de laboratórios operantes, entre outras. Tal pesquisa tem produzido um corpo de procedimentos para manter e alterar comportamento que está encontrando aplicação numa variedade de áreas. Os procedimentos desenvolvidos estão também sendo usados numa análise experimental que gera conhecimento teórico e relações funcionais sobre o comportamento. Embora os procedimentos tenham sido freqüentemente identificados com animais simples engajados em comportamentos simples sob condições altamente restritas, os métodos de análise e controle estão agora sendo extendidos com sucesso para comportamentos humanos muito complexos em interação social sob condições variáveis. (Goldiamond \& Dyrud, 1968, p. 55)

A complementariedade entre a Análise Experimental do Comportamento e a Análise Comportamental Aplicada é também defendida por Ferster (1979). Segundo este autor,

ambas podem estar preocupadas com os mesmos itens de conduta com objetivos diferentes. O profissional é obrigado a se engajar em qualquer problema que o cliente trouxer. Sua tarefa principal é melhorar a angústia. Os profissionais se envolvem totalmente e geralmente não podem ser responsáveis por provar o que foi realizado. Por outro lado, psicólogos experimentais escolhem seu próprio chão, sendo solicitados apenas a olhar objetivamente e com clareza conceitual e a comunicar nos termos da ciência natural. (p. 23)

Portanto, no campo da Análise do Comportamento, parece haver um reconhecimento de que os interesses e atividades dos cientistas básicos e dos analistas comportamentais aplicados são diferentes porém complementares. Apesar disso, não se deve cair na tentação de pensar ingenuamente que pesquisadores aplicados e prestadores de serviço são ou deveriam ser, de fato, companheiros de trabalho em algum empreendimento filantrópico comum.

Por outro lado, também não se pode considerar negativa toda e qualquer combinação de atividades de pesquisa e prestação de serviço no campo da Análise do Comportamento. Cada caso precisa ser examinado individualmente pois, desde que se busque encarar funcionalmente as relações recíprocas entre comportamento e ambiente, pesquisa e prestação de serviço podem ser combinadas. Na verdade, há imensas lacunas no conhecimento existente sobre essas relações e provavelmente muitas maneiras diferentes de produzi-lo. De qualquer modo, o esclarecimento das relações entre comportamento e ambiente dependerá do esforço conjunto daqueles que se dedicam à produção do conhecimento, à prestação de serviços e, sobretudo, daqueles que conseguirem produzir conhecimento enquanto intervêm na situação natural. 


\section{Referências}

Azrin, N. H. (1977). A strategy for applied research: Learning based but outcome oriented. American Psychologist, 32, 140-149.

Baer, D. M., Wolf, M. M. \& Risley, T. R. (1968). Some current dimensions of applied behavior analysis. Journal of Applied Behavior Analysis, 1, 91-97.

Bennett, M. L. (1987). Radical behaviorism analysis of interpretive verbal behavior in applied settings. Tese de Doutorado não-publicada, Programa de PósGraduação em Psicologia, University of Nevada. Nevada, Reno.

Birnbrauer, J. S. (1979). Applied behavior analysis, service and the acquisition of knowledge. The Behavior Analyst, 2, 15-21.

Deitz, S. M. (1978). Current status of applied behavior analysis: Science versus technology. American Psychologist, 33, 805-814.

Deitz, S. M.(1983). Two correct definitions of "Applied". The Behavior Analyst, 6, 105-106.

Ferreira, A. B. H. (1986). Novo dicionário da Lingua Portuguesa (2 ed. rev.). Rio de Janeiro: Nova Fronteira.

Ferster (1979). A laboratory model of Psychotherapy: The boundary between clinical practice and experimental psychology. Em P. Sjödén, S. Bates \& W. Dockens (Orgs.), Trends in behavior therapy (pp. 23-38). London: Academic Press.

Goldiamond, I. (1965). Training in behavior modification. Em APA (Org.), Preconference materials conference on the professional preparation of clinical psychologists (pp. 56-58). Washington, D.C.: American Psychological Association.

Goldiamond, I. \& Dyrud, J. (1968). Some applications and implications of Behavioral Analysis for Psychotherapy. Research in Psychotherapy, 3, 54-89.

Hayes, S. C. (1998). Construindo uma relação útil entre ciência aplicada e básica na terapia comportamental. Boletim Informativo da ABPMC, 15, 4-7.

Hayes, S. C., Rincover, A. \& Solnick, J. V. (1980). The technical drift of applied behavior analysis. Journal of Applied Behavior Analysis, 13, 275-285.
Johnston, J. M. (1996). Distinguishing between applied research and practice. The Behavior Analyst, 19, 35-47.

Luna, S. V. (1997). O terapeuta é um cientista? Em R. A. Banaco (Org.), Sobre comportamento e cognição: Aspectos teóricos, metodológicos e de formação em análise do comportamento e terapia cognitivista (pp. 305-313). São Paulo: Arbytes.

Pierce, W. D. \& Epling, W. F. (1980). What happened to analysis in applied behavior analysis? The Behavior Analyst, 3, 1-9.

Poling, A., Picker, M. \& Grossett, D. (1981). The schism between expe- rimental and applied behavior analysis: Is it real and who cares? The Behavior Analyst, 4, 93-102.

Ryle, G. (1993). Dilemas (A. Cabral, Trad.). São Paulo: Martins Fontes. (Original Publicado em 1954)

Skinner, B. F. (1985). Ciência e comportamento bumano (J. C. Todorov \& R. Azzi, Trad.) ( $6^{2}$ ed.). São Paulo: Martins Fontes. (Original publicado em 1953)

Watson, J. B. (1913). Psychology as the behaviorist views it. Psychological Review, 20, 158-177.

Wittgenstein, L. (1987). Investigações filosóficas (J. C. Bruni, Trad.). Lisboa: Fundação Calouste Gulbenkian. (Original publicado em 1953)

Recebido: 14/06/2002

$1^{a}$ Revisão: $19 / 08 / 2002$

Última Revisão: 10/12/2002

Aceite Final: 20/01/2003

Sobre a autora

Silvia Canaan-Oliveira é Psicóloga (Terapeuta Comportamental), Mestre em Musicoterapia pela University of Missouri - Kansas City (EUA), Doutora em Psicologia pela Universidade de Brasília (UnB). É Professora da Universidade Federal do Pará. 